ACTA SCIENTIFIC MEDICAL SCIENCES (ISSN: 2582-0931)

Volume 3 Issue 11 November 2019

Short Communication

\title{
Role of EKOS® in Management of Pulmonary Embolism
}

\author{
Kashmala Khan ${ }^{\mathbf{1}}$ and Salim R Surani ${ }^{2 *}$ \\ ${ }^{1}$ PGY-1 Internal Medicine Resident, Corpus Christi Medical Center, Corpus Christi, Texas, USA \\ ${ }^{2}$ Adjunct Clinical Professor of Medicine, Texas A\&M University, Health Science Center, College of Medicine, Texas, USA \\ *Corresponding Author: Salim Surani, Adjunct Clinical Professor of Medicine, Texas A\&M University, Health Science Center, College of \\ Medicine, Texas, USA.
}

Received: October 03, 2019; Published: October 10, 2019

DOI: $10.31080 / A S M S .2019 .03 .0434$

Pulmonary embolism (PE) is a form of venous thromboembolism that has a devastating and potentially fatal clinical course with an array of symptoms and presentation that has become a cause of great concern to health professionals [1-3]. In the United Sates, the incidence of venous thromboembolism ranges from about 900,000 [1] which includes both Deep Vein Thrombosis and PE, with PE accounting for about 150,000-250,000 hospitalizations, and about 60,000-100,000 deaths annually [1]. Early diagnosis, treatment, and risk stratification is imperative and can be lifesaving. Overall mortality is high, with sudden death being the initial symptom for about 25\% of patients [3]. Pulmonary Embolism is classified based on pattern of presentation (acute, subacute or chronic), presence or absence of hemodynamic stability and anatomic location (saddle, lobar, segmental, sub segmental). Patients with Massive PE have hemodynamic instability with systolic blood pressure $<90 \mathrm{mmHg}$, and signs of shock. Sub massive PE patients are hemodynamically stable with no signs of shock. Patients are seen to have right ventricular dysfunction, with increased Right Ventricle (RV)/Left Ventricle (LV) ratios [2]. Venous thromboembolism (VTE) especially pulmonary embolism imposes a huge burden on the healthcare system. The annual cost of VTE ranges from $\$ 5-10$ billion dollars [5].

Treatment options takes into consideration patterns of presentation and the hemodynamic status of the patient. After confirmation with clinical assessment and diagnostic testing treatment is initiated without delay. FDA approved treatments include Anticoagulation, Reperfusion therapy with Thrombolytics or Emblolectomy (surgical vs Catheter guided). There has been much debate about the risks and benefits of thrombolytic therapy and the decision is tailored to each specific patient [6].
Thrombolytic therapy can be either administered systemically or via Catheter Directed Interventions (CDI) [7]. CDI includes catheter induced thrombus fragmentation (uses manual fragmentation by rotation of pigtail catheter in the pulmonary artery), rheolytic thrombectomy (uses saline jet), catheter mediated thromboembolus aspiration and catheter directed thrombolysis via infusion catheters [7]. One such catheter-based modality that uses thrombolysis via infusion catheters is the EkoSonic Endovascular System or EKOS ${ }^{\circledR}$ [8]. This has been approved by the FDA for treatment of acute massive or sub massive pulmonary emboli and is also available to treat deep vein thrombosis [8]. The rationale behind using this particular therapy in PE patients is to use shorter infusions and lower doses of thrombolytic therapy thus reducing the increased bleeding risks associated with their use. EKOS ${ }^{\circledR}$ works by using ultrasound energy waves with high frequency and low power to help make the clot more permeable to the thrombolytic therapy and thus helps to dissolve it [8].

The SEATTLE II trial is a prospective, single-arm study which enrolled 150 patients with massive and sub massive PE. It successfully demonstrated significant improvement in Right Ventricular strain with reduction in $\mathrm{RV} / \mathrm{LV}$ ratio, decrease in pulmonary hypertension and angiographic obstruction post procedure [9]. The ULTIMA trial [10] compares the efficacy of EKOS $®$ and thrombolytic therapy in combination with Heparin to the use of Heparin therapy alone in a total of 59 patients. It successfully established that a fixed dose of the combination therapy was superior to using anticoagulation alone in improving right ventricular dysfunction in patients with acute PE. The OPTALYSE trial [11] is a randomized cohort study that focused on 101 patients with sub massive PE. This 
trial used varying doses and duration of therapy and found that low dose, low duration of thrombolytic therapy is just as effective with using EKOS $^{\circledR}$ as other regimens (studied in SEATTLE II and ULTIMA).

There are demonstrated benefits of using the EKOS ${ }^{\circledR}$ ultrasound facilitated catheter directed thrombolysis. It is evident that there is a decrease in $\mathrm{RV} / \mathrm{LV}$ ratio, with improvement of right ventricular function with this treatment modality. With decreased doses and duration of thrombolytic therapy, patients potentially have a lower risk of bleeding which is the most feared complication of this treatment. There are limitations, as this is an area that requires much more research and it is a treatment modality that is far from being the gold standard. Some physicians are hesitant and question the benefit of this procedure in patients with massive PE. One reason for this is the severity and dramatic presentation of massive $\mathrm{PE}$ which is time sensitive and requires prompt recognition and immediate treatment. There may not always be enough time to go through the protocol and preparation required for these procedures, and in such instance administration of systemic thrombolytics may seem like the better option. In addition, there is no consensus on a single low dose of a Thrombolytic agent, different studies have used various doses [9-11]. There is still need for long term follow up with these patients to assess for recurrence of thromboembolic disease (recurrent PE, Chronic Thromboembolic Pulmonary Hypertension etc.) and long-term bleeding complications. Studies have yet to demonstrate the long-term advantages or adverse effects EKOS $^{\circledR}$ and catheter based thrombolytics can potentially have when compared to systemic thrombolysis. Such studies would have to follow patients for at least 5 years or more, and have a standard follow up protocol, which may prove difficult as patients are usually lost to follow up. There are clear advantages to using ultrasound facilitated catheter directed thrombolytic therapy. It should be used in patients with sub massive PE due to lower exposure to thrombolytics. However, there is a lack of dual arm randomized trials that compare catheter directed thrombolytic therapy with systemic thrombolysis, which prevents this treatment modality to be used more widely.

\section{Bibliography}

1. Rivera-Lebron B., et al. "Diagnosis, Treatment and Follow Up of Acute Pulmonary Embolism: Consensus Practice from the PERT Consortium". Clinical and Applied Thrombosis/Hemostasis 25 (2019):1076029619853037.
2. Beckman MG., et al. "Venous thromboembolism: a public health concern". American Journal of Preventive Medicine 38(2010): S495-501.

3. Kalani C., et al. "The innovations in pulmonary hypertension pathophysiology and treatment: What are our options?" Current Respiratory Medicine Reviews 14 (2018): 189-203.

4. CDC: www.cdc.gov ; Data and Statistics on venous thromboembolism.

5. Grosse SD., et al. "The economic burden of incident venous thromboembolism in the United States: A review of estimated attributable healthcare costs". Thromb Research 137 (2016): 3-10.

6. Martin C., et al. "Systemic Thrombolysis for Pulmonary Embolism: A Review". P T 41 (2016): 770-775.

7. Zarghouni., et al. "Catheter-directed interventions for pulmonary embolism". Cardiovascular diagnosis and therapy 6.6 (2016): 651-661.

8. Garcia Mark J. "Endovascular Management of Acute Pulmonary Embolism Using the Ultrasound-Enhanced EkoSonic System". Seminars in Interventional Radiology 32.4 (2015): 384-387.

9. Piazza G., et al. "A Prospective, Single-Arm, Multicenter Trial of Ultrasound-Facilitated, Catheter-Directed, Low-Dose Fibrinolysis for Acute Massive and Submassive Pulmonary Embolism: The SEATTLE II Study". JACC Cardiovascular Intervention 8(2015):1382-1392.

10. Kucher N., et al. "Randomized, controlled trial of ultrasoundassisted catheter-directed thrombolysis for acute intermediate-risk pulmonary embolism". Circulation 129 (2014): 479486.

11. Tapson VF., et al. "A Randomized Trial of the Optimum Duration of Acoustic Pulse Thrombolysis Procedure in Acute Intermediate-Risk Pulmonary Embolism: The OPTALYSE PE Trial". JACC: Cardiovascular Interventions 11 (2018):1401-1410.

\section{Volume 3 Issue 11 November 2019 (c) All rights are reserved by Kashmala Khan and Salim $\mathbf{R}$} Surani. 